\title{
Pseudo-elementary generalized triangle groups
}

\author{
Gerald Williams
}

(Communicated by A. Yu. Olshanskii)

\begin{abstract}
A generalized triangle group is a group that can be presented in the form $\Gamma=\left\langle x, y \mid x^{l}=y^{m}=w(x, y)^{n}=1\right\rangle$ where $l, m, n \in\{2,3,4, \ldots\} \cup\{\infty\}$ and $w(x, y)$ is an element of the free product $\left\langle x, y \mid x^{l}=y^{m}=1\right\rangle$ involving both $x$ and $y$. A homomorphism $\phi: \Gamma \rightarrow G$ is said to be essential if $\phi(x), \phi(y), \phi(w(x, y))$ have orders $l, m, n$ respectively. Every generalized triangle group $\Gamma$ admits an essential representation to $\operatorname{PSL}(2, \mathbb{C})$. In most cases there will be such a representation with infinite or non-elementary image.

Vinberg and Kaplinsky say that $\Gamma$ is pseudo-finite if the image of any essential representation $\Gamma \rightarrow \operatorname{PSL}(2, \mathbb{C})$ is finite and they have obtained a partial classification of such groups. Extending this concept, we call $\Gamma$ pseudo-elementary if the image of any essential representation $\Gamma \rightarrow \operatorname{PSL}(2, \mathbb{C})$ is elementary. In this paper we classify the pseudo-elementary generalized triangle groups $\Gamma$ with $n \geqslant 3$ and obtain partial results in the case $n=2$.
\end{abstract}

\section{Introduction}

We consider groups that can be presented in the form

$$
\Gamma=\left\langle x, y \mid x^{l}=y^{m}=w(x, y)^{n}=1\right\rangle
$$

where $l, m, n \in\{2,3,4, \ldots\} \cup\{\infty\}, l \leqslant m,(l, m) \neq(2,2)$, where

$$
w(x, y)=x^{\alpha_{1}} y^{\beta_{1}} \ldots x^{\alpha_{k}} y^{\beta_{k}}
$$

with

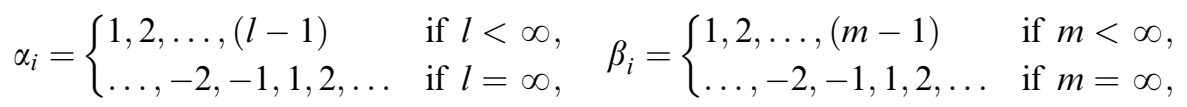

and where $w(x, y)$ is not a proper power. Such groups are called generalized triangle groups, and these have been studied for a variety of algebraic, geometric, and topological reasons. One problem concerns the Tits alternative. A group is said to satisfy 
the Tits alternative if it either contains a non-abelian free subgroup or has a soluble subgroup of finite index. A pair of elements $\{u, v\}$ of a group $G$ is called a ReeMendelsohn pair if there exists some integer $N$ such that $\left\langle u^{N}, v^{N}\right\rangle$ is free of rank 2. Clearly if $G$ has a Ree-Mendelsohn pair, then $G$ contains a non-abelian free subgroup.

Rosenberger has conjectured that every generalized triangle group satisfies the Tits alternative. (See [9] or [13] for a survey of this problem, and [3], [2], [15] for more recent results.) Related to this conjecture is the question of which generalized triangle groups contain Ree-Mendelsohn pairs.

A homomorphism $\phi: \Gamma \rightarrow G$ (for some group $G$ ) is called essential if $\phi(x), \phi(y)$, $\phi(w(x, y))$ have orders $l, m, n$ respectively. Every generalized triangle group $\Gamma$ admits an essential representation to $\operatorname{PSL}(2, \mathbb{C})$ (see [1], [7], [10]), and admits an essential representation to $\operatorname{SO}(3)$ (see [4]). A subgroup of $\operatorname{PSL}(2, \mathbb{C})$ is called elementary if it contains no non-abelian free subgroup. In most cases a generalized triangle group $\Gamma$ will admit an essential representation to $\operatorname{PSL}(2, \mathbb{C})$ with non-elementary image, and thus $\Gamma$ will contain a non-abelian free subgroup. Moreover, by [8, Theorem 8], in these cases $\Gamma$ will contain a Ree-Mendelsohn pair.

There are, however, generalized triangle groups $\Gamma$ that contain non-abelian free subgroups but whose essential representations to $\operatorname{PSL}(2, \mathbb{C})$ all have elementary image. Similarly, there exist infinite generalized triangle groups $\Gamma$ whose essential representations to $\operatorname{PSL}(2, \mathbb{C})$ all have finite image. Vinberg and Kaplinsky [12] have termed a generalized triangle group $\Gamma$ pseudo-finite if the image of any essential representation $\Gamma \rightarrow \operatorname{PSL}(2, \mathbb{C})$ is finite. We likewise call $\Gamma$ pseudo-elementary if the image of any essential representation $\Gamma \rightarrow \operatorname{PSL}(2, \mathbb{C})$ is elementary. Vinberg and Kaplinsky have classified the pseudo-finite generalized triangle groups $\Gamma$ for $n \geqslant 3$, and for $n=2, k \leqslant 3$.

In this paper we carry out similar investigations for pseudo-elementary generalized triangle groups. We classify all such groups for $k=1$ (Section 4 ), for $n \geqslant 3$ (Section 5), and provide partial results in the case $n=2$ (Sections 3 and 6). For $n \geqslant 3$ the classification of the pseudo-elementary generalized triangle groups was already implicit in the papers of Fine, Rosenberger, et al. [6], [8], [9], [10], [13], [14], and for $n=2$ the partial classification was already implicit in [16], but never formally stated. Here we codify the results and simplify the methods.

\section{Preliminaries}

Throughout this paper $\Gamma$ will refer to the generalized triangle group defined by the presentation (1). There are some obvious transformations of this presentation that lead to an isomorphic group. We shall take such transformations into account as follows. Two cyclically reduced words $w(x, y), w^{\prime}(x, y) \in\left\langle x, y \mid x^{l}=y^{m}=1\right\rangle$ are said to be equivalent if $w(x, y)$ can be transformed to $w^{\prime}(x, y)$ by a sequence of moves of the form:

(1) cyclic permutation;

(2) inversion; 
(3) automorphism of $\left\langle x \mid x^{l}\right\rangle$ (if $\left.l<\infty\right)$ or $\left\langle y \mid y^{m}\right\rangle$ (if $m<\infty$ );

(4) interchanging $x$ and $y$ (if $l=m$ ).

If two words $w(x, y), w^{\prime}(x, y)$ are equivalent then the corresponding generalized triangle groups are said to be equivalent. Equivalent groups are isomorphic, and so for this reason we consider generalized triangle groups only up to equivalence, although we will not explicitly state this in each result.

We now recall some facts concerning the groups $\operatorname{SL}(2, \mathbb{C})$ and $\operatorname{PSL}(2, \mathbb{C})$. For a matrix $A \in \mathrm{SL}(2, \mathbb{C})$ we shall denote by $[A]$ the corresponding projective matrix $\{A,-A\}$ of $\operatorname{PSL}(2, \mathbb{C})$. The element $[A]$ has order $N \geqslant 2$ if and only if $\operatorname{tr} A=2 \cos (r \pi / N)$ where $(r, N)=1$. Let $A, B \in \operatorname{SL}(2, \mathbb{C})$. The group $\langle[A],[B]\rangle$ is said to be reducible if and only if $\operatorname{tr}[A, B]=2$ (where $[A, B]=A B A^{-1} B^{-1}$ ). Note that

$$
\operatorname{tr}[A, B]=(\operatorname{tr} A)^{2}+(\operatorname{tr} B)^{2}+(\operatorname{tr} A B)^{2}-(\operatorname{tr} A)(\operatorname{tr} B)(\operatorname{tr} A B)-2 .
$$

The subgroup $\langle[A],[B]\rangle$ of $\operatorname{PSL}(2, \mathbb{C})$ is elementary if and only if it is finite, infinite dihedral, or reducible. The finite subgroups of $\operatorname{PSL}(2, \mathbb{C})$ are the alternating groups $A_{4}, A_{5}$, the symmetric group $S_{4}$, the finite dihedral groups, and the finite cyclic groups (see for example [5, pp. 84-86]). If $\langle[A],[B]\rangle$ is a finite cyclic group then $A$ and $B$ commute, so that $[A, B]=I$ and $\operatorname{tr}[A, B]=2$. The group $\langle[A],[B]\rangle$ is dihedral if and only if two or more of $[A],[B],[A B]$ are of order 2 . We record the above facts:

Theorem 1 (see [5]). Let $A, B \in \operatorname{SL}(2, \mathbb{C})$. Then $\langle[A],[B]\rangle \leqslant \operatorname{PSL}(2, \mathbb{C})$ is elementary if and only if one or more of the following holds:

(1) $\langle[A],[B]\rangle$ is isomorphic to $A_{4}, S_{4}$ or $A_{5}$;

(2) $(\operatorname{tr} A)^{2}+(\operatorname{tr} B)^{2}+(\operatorname{tr} A B)^{2}-(\operatorname{tr} A)(\operatorname{tr} B)(\operatorname{tr} A B)-4=0$;

(3) at least two of $\operatorname{tr} A, \operatorname{tr} B, \operatorname{tr} A B$ are equal to zero.

Every generalized triangle group admits an essential representation to $\operatorname{PSL}(2, \mathbb{C})$ (see [1], [7], [10]), as we now describe. Let $X, Y$ be elements of $\operatorname{SL}(2, \mathbb{C})$ such that $[X]$, $[Y]$ have orders $l, m$ respectively. We shall obtain necessary and sufficient conditions on $X, Y$ for the map $\rho: \Gamma \rightarrow \operatorname{PSL}(2, \mathbb{C})$ given by $x \mapsto[X], y \mapsto[Y]$ to define an essential representation.

If $n=\infty$ then $\Gamma$ is not pseudo-elementary (see Section 3), and so we may assume that $n<\infty$. If $\rho$ is essential then the element $w([X],[Y])$ has order $n$ so that $\operatorname{tr} w(X, Y)=2 \cos (r \pi / n)$ where $(r, n)=1$. Now $\operatorname{tr} w(X, Y)$ is a polynomial in $\operatorname{tr} X$, $\operatorname{tr} Y$ and $\lambda:=\operatorname{tr} X Y$, of degree $k$ in $\lambda$. We call this the trace polynomial and (suppressing $\operatorname{tr} X, \operatorname{tr} Y$ in the notation) write $\tau_{w}(\lambda):=\operatorname{tr} w(X, Y)$. Thus $\rho$ defines an essential representation if and only if $\lambda$ is a root of

$$
\tau_{w}(\lambda)=2 \cos (r \pi / n)
$$

where $(r, n)=1$. 
If $l, m<\infty$ then $\operatorname{tr} X=2 \cos (p \pi / l), \operatorname{tr} Y=2 \cos (q \pi / m)$ where $(p, l)=(q, m)=1$. But, up to equivalence, $\Gamma$ is unchanged by applying an automorphism of $\left\langle x \mid x^{l}\right\rangle$ or $\left\langle y \mid y^{m}\right\rangle$ and so we may assume that $p=q=1$. Moreover, we can choose $X, Y$ to be any elements of $\operatorname{SL}(2, \mathbb{C})$ with these traces. Case 2 of Theorem 1 is then equivalent to $\lambda=2 \cos (\pi / l \pm \pi / m)$.

We wish to single out the cases where the generalized triangle group $\Gamma$ can admit essential representations to any of the finite subgroups $A_{4}, S_{4}, A_{5}$ of $\operatorname{PSL}(2, \mathbb{C})$. Suppose that $[A],[B]$ have orders $l, m$, respectively where $l \leqslant m$. Then $[A],[B]$ can generate $A_{4}, S_{4}$, or $A_{5}$ only if

$$
(l, m)=(2,3),(2,4),(2,5),(3,3),(3,4),(3,5),(4,4), \text { or }(5,5)
$$

(see [12]). We shall say that $\Gamma$ is exceptional if $(l, m)$ is one of the pairs listed in (3), and non-exceptional otherwise.

If $\Gamma$ is non-exceptional then the roots of $\tau_{w}(\lambda)$ are either $\lambda=0$ or $\lambda=2 \cos (\pi / l \pm \pi / m)$. In the latter case there also exists an essential representation of $\Gamma$ to a cyclic subgroup of $\operatorname{PSL}(2, \mathbb{C})$, given by $x \mapsto[X], y \mapsto[Y]$ where

$$
X=\left(\begin{array}{cc}
e^{i \pi / l} & 0 \\
0 & e^{-i \pi / l}
\end{array}\right), \quad Y=\left(\begin{array}{cc}
e^{i \pi / m} & 0 \\
0 & e^{-i \pi / m}
\end{array}\right)
$$

\section{Infinite exponents}

In this section we consider the case when at least one of $l, m, n$ is equal to $\infty$. If $n=\infty$ then $\Gamma=\left\langle x, y \mid x^{l}=y^{m}=1\right\rangle$, which is isomorphic to a Fuchsian group of signature $(0: l, m, \infty)$ and so there is a faithful (and hence essential) representation $\Gamma \rightarrow \mathbb{Z}_{l} * \mathbb{Z}_{m} \leqslant \operatorname{PSL}(2, \mathbb{C})$. Since $(l, m) \neq(2,2)$, the group $\Gamma$ is not pseudoelementary. If $l=m=\infty$ then, by Ree and Mendelsohn [11], $\Gamma$ is not pseudoelementary.

Suppose then that $l, n<\infty$ and $m=\infty$. The results [9, Lemmas 1-3] (or [13, Lemmas 7.3.3.1-7.3.3.3]) cover most cases:

Theorem 2 ([9]). Let $l, n<\infty, m=\infty$ and suppose that one of the following holds:

(1) $(l, n) \neq(2,2)$;

(2) $(l, n)=(2,2)$ and either

(a) $k$ is even or

(b) $k \geqslant 3$ is odd and $\sum_{i=1}^{k} \beta_{i} \neq 0$.

Then $\Gamma$ is not pseudo-elementary.

If $(l, n)=(2,2)$ and $k$ is odd then $\Gamma$ can be pseudo-elementary in very many cases. We consider the pseudo-elementary groups $\Gamma$ for $k=1,3,5$.

Let $X, Y$ be the matrices in $\operatorname{SL}(2, \mathbb{C})$ defined by 


$$
X=\left(\begin{array}{cc}
0 & 1 \\
-1 & 0
\end{array}\right), \quad Y=\left(\begin{array}{cc}
z & 0 \\
\lambda & z^{-1}
\end{array}\right),
$$

where $z, \lambda \in \mathbb{C}$ and $z+z^{-1} \neq 2 \cos (r \pi / N)$ for any $N \geqslant 2,(r, N)=1$. Then $\operatorname{tr} X=0$, $\operatorname{tr} Y=z+z^{-1}$, and $\operatorname{tr} X Y=\lambda$. The conditions on $z$ are necessary and sufficient for $[Y]$ to be of infinite order. Theorem 1 implies that $\Gamma$ is pseudo-elementary if and only if the roots of the trace polynomial $\tau_{w}(\lambda)$ either are $\lambda=0$ or satisfy $\lambda^{2}=-\left(z-z^{-1}\right)^{2}$.

Note that if $z \neq \pm 1$ then

$$
Y^{\beta}=\left(\begin{array}{cc}
z^{\beta} & 0 \\
\lambda p_{\beta}(z) & z^{-\beta}
\end{array}\right)
$$

where

$$
p_{\beta}(z)=\left(z^{\beta}-z^{-\beta}\right) /\left(z-z^{-1}\right),
$$

and the leading coefficient of $\tau_{w}(\lambda)=\operatorname{tr} w(X, Y)$ is given by $c=\prod_{i=1}^{k} p_{\beta_{i}}(z)$.

If $k=1$ the trace polynomial is given by $\tau_{w}(\lambda)=c \lambda$ (if $z \neq \pm 1$ ) or $\tau_{w}(\lambda)=\beta_{1} \lambda$ (if $z= \pm 1$ ), with root $\lambda=0$. Thus $\Gamma$ is pseudo-elementary. Consider the case $k=3$.

Proposition 3. $\Gamma$ is pseudo-elementary if and only if $\beta_{1}+\beta_{2}+\beta_{3}=0$.

Proof. If $z \neq \pm 1$, a calculation shows that the trace polynomial

$$
\tau_{w}(\lambda)=\operatorname{tr}\left(X Y^{\beta_{1}} X Y^{\beta_{2}} X Y^{\beta_{3}}\right)
$$

is given by

$$
\tau_{w}(\lambda)=c \lambda\left(\lambda^{2}-\frac{\left(z-z^{-1}\right)^{2}}{\prod_{i=1}^{3}\left(z^{\beta_{i}}-z^{-\beta_{i}}\right)} P(z)\right)
$$

where

$$
P(z)=\left(z^{\beta_{1}-\beta_{2}+\beta_{3}}-z^{-\beta_{1}+\beta_{2}-\beta_{3}}\right)+\left(z^{\beta_{1}+\beta_{2}-\beta_{3}}-z^{-\beta_{1}-\beta_{2}+\beta_{3}}\right)+\left(z^{-\beta_{1}+\beta_{2}+\beta_{3}}-z^{\beta_{1}-\beta_{2}-\beta_{3}}\right) .
$$

By Theorem 2 we may assume that $\beta_{1}+\beta_{2}+\beta_{3}=0$, so that

$$
P(z)=-\prod_{i=1}^{3}\left(z^{\beta_{i}}-z^{-\beta_{i}}\right)
$$

and hence

$$
\tau_{w}(\lambda)=c \lambda\left(\lambda^{2}+\left(z-z^{-1}\right)^{2}\right)
$$

which is equal to zero if and only if $\lambda=0$ or $\lambda^{2}=-\left(z-z^{-1}\right)^{2}$.

If $z= \pm 1$ then $\tau_{w}(\lambda)= \pm \beta_{1} \beta_{2} \beta_{3} \lambda^{3}$ which is equal to zero if and only if $\lambda=0$. 
Consider now the case $k=5$. By Theorem 2, if $\Gamma$ is pseudo-elementary then $\sum_{i=1}^{5} \beta_{i}=0$. Suppose first that $z \neq \pm 1$. A calculation shows that the trace polynomial is given by

$$
\operatorname{tr} w(X, Y)=c \lambda\left(\lambda^{4}+\left(1+\frac{Q(z)}{\prod_{i=1}^{5}\left(z^{\beta_{i}}-z^{-\beta_{i}}\right)}\right)\left(z-z^{-1}\right)^{2} \lambda^{2}+\frac{\left(z-z^{-1}\right)^{4} Q(z)}{\prod_{i=1}^{5}\left(z^{\beta_{i}}-z^{-\beta_{i}}\right)}\right)
$$

where

$$
Q(z)=\left(z^{B_{1}}-z^{-B_{1}}\right)+\left(z^{B_{2}}-z^{-B_{2}}\right)+\left(z^{B_{3}}-z^{-B_{3}}\right)+\left(z^{B_{4}}-z^{-B_{4}}\right)+\left(z^{B_{5}}-z^{-B_{5}}\right)
$$

with

$$
\begin{aligned}
& B_{1}=\beta_{1}+\beta_{2}-\beta_{3}+\beta_{4}-\beta_{5}, \\
& B_{2}=\beta_{1}-\beta_{2}+\beta_{3}-\beta_{4}+\beta_{5}, \\
& B_{3}=\beta_{1}-\beta_{2}+\beta_{3}+\beta_{4}-\beta_{5}, \\
& B_{4}=-\beta_{1}+\beta_{2}-\beta_{3}+\beta_{4}+\beta_{5}, \\
& B_{5}=-\beta_{1}+\beta_{2}+\beta_{3}-\beta_{4}+\beta_{5} .
\end{aligned}
$$

If $\Gamma$ is pseudo-elementary then the restrictions on the roots of the trace polynomial mean that $\tau_{w}(\lambda)$ takes one of three forms:

$$
\begin{aligned}
& \tau_{w}(\lambda)=c \lambda^{5} \\
& \tau_{w}(\lambda)=c \lambda^{3}\left(\lambda^{2}+\left(z-z^{-1}\right)^{2}\right) ; \\
& \tau_{w}(\lambda)=c \lambda\left(\lambda^{4}+2\left(z-z^{-1}\right)^{2} \lambda^{2}+\left(z-z^{-1}\right)^{4}\right) .
\end{aligned}
$$

The first form does not arise as it requires both $1+Q(z) / \prod_{i=1}^{5}\left(z^{\beta_{i}}-z^{\beta_{i}}\right)=0$ and $Q(z)=0$, which is impossible. Consider the second form.

Proposition 4. Suppose that $z \neq \pm 1$. Then $\tau_{w}(\lambda)=c \lambda^{3}\left(\lambda^{2}+\left(z-z^{-1}\right)^{2}\right)$ if and only if $\left(\beta_{1}, \beta_{2}, \beta_{3}, \beta_{4}, \beta_{5}\right)$ is of one of the following forms, where $a, b \in\{\ldots,-2,-1,1,2, \ldots\}$ :

(1) $(a, b, b-a,-a-b, a-b)$ where $b \neq \pm a$;

(2) $(a, b,-2 a+b,-b, a-b)$ where $b \neq a, b \neq 2 a$;

(3) $(a,-a-2 b,-a, b, a+b)$ where $b \neq-a, a \neq-2 b$;

(4) $(a,-a-2 b,-a-b, b, a+2 b)$ where $a \neq-b, a \neq-2 b$.

Proof. The polynomial $\tau_{w}(\lambda)$ takes this form if and only if $Q(z)=0$ for all allowed $z$, which occurs if and only if $\left\{B_{1}, B_{2}, B_{3}, B_{4}, B_{5}\right\}=\left\{-B_{1},-B_{2},-B_{3},-B_{4},-B_{5}\right\}$. Note 
that each $\beta_{i} \neq 0$. A straightforward combinatorial argument shows the following: $B_{1} \neq-B_{2} ; B_{1} \neq-B_{5} ; B_{1}=-B_{1}$ if and only if Case 1 holds, in which case $B_{2}=-B_{3}$, $B_{4}=-B_{5} ; B_{1}=-B_{3}$ if and only if Case 2 or Case 3 holds, and then $B_{2}=0$, $B_{4}=-B_{5}$ or $B_{2}=-B_{5}, B_{4}=0$, respectively; $B_{1}=-B_{4}$ if and only if Case 4 holds, in which case $B_{2}=-B_{5}, B_{3}=0$.

$$
\begin{aligned}
& \text { If } z= \pm 1 \text { then the trace polynomial is given by } \\
& \tau_{w}(\lambda)= \pm\left(\beta_{1} \beta_{2} \beta_{3} \beta_{4} \beta_{5} \lambda^{5}-\left(\beta_{1} \beta_{2} \beta_{3}+\beta_{2} \beta_{3} \beta_{4}+\beta_{3} \beta_{4} \beta_{5}+\beta_{4} \beta_{5} \beta_{1}+\beta_{5} \beta_{1} \beta_{2}\right) \lambda^{3}\right) .
\end{aligned}
$$

In each of the cases obtained in Proposition 4 it is easy to check that the coefficient of $\lambda^{3}$ equates to zero, and thus $\Gamma$ is pseudo-elementary precisely in these cases.

The third form of $\tau_{w}(\lambda)$ arises if and only if $\prod_{i=1}^{5}\left(z^{\beta_{i}}-z^{-\beta_{i}}\right)=Q(z)$ for all allowed $z$. We have not been able to classify the groups $\Gamma$ corresponding to this case. For the remainder of the paper we assume that $l, m, n<\infty$.

\section{The case $k=1$}

Theorem 5. Suppose that $k=1$ and $\alpha_{1}\left|l, \beta_{1}\right| m$, and set $\kappa=\alpha_{1} / l+\beta_{1} / m+1 / n$.

(1) If $\kappa>1$ then $\Gamma$ is pseudo-elementary if and only if $\Gamma$ is pseudo-finite.

(2) If $\kappa=1$ then $\Gamma$ is pseudo-elementary but not pseudo-finite.

(3) If $\kappa<1$ then $\Gamma$ is not pseudo-elementary.

Proof. Note that the trace polynomial has precisely one root.

(1) Consider a homomorphism $\rho: \Gamma \rightarrow \mathbb{Z}_{l m n}$. If $\rho$ is essential, then $\rho(x)=p m n$, $\rho(y)=q \ln$ where $(p, \operatorname{lm} n)=(q, \operatorname{lm} n)=1$. By applying an automorphism to $\left\langle x \mid x^{l}\right\rangle$ or $\left\langle y \mid y^{m}\right\rangle$ we may assume that $p=q=1$. If $\rho$ is essential then $\rho\left(x^{\alpha_{1}} y^{\beta_{1}}\right)$ is of order $n$, i.e. we can write

$$
\alpha_{1} m n+\beta_{1} \ln =r \operatorname{lm}
$$

with $(r$, lmn $)=1$. Since $\kappa>1$, we have $\left\{l / \alpha_{1}, m / \beta_{1}, n\right\}=\{3,3,2\},\{3,4,2\},\{3,5,2\}$, or $\{2,2, j\}$ where $j \geqslant 2$. It is then easy to show that $(5)$ has no solution in these cases, and hence $\Gamma$ does not admit any essential representation to a cyclic group. Thus the numbers $2 \cos (\pi / l \pm \pi / m)$ do not occur as roots of $\tau_{w}(\lambda)$ and Case 2 of Theorem 1 cannot arise. In Case $1, \lambda$ corresponds to an essential representation to a finite subgroup of $\operatorname{PSL}(2, \mathbb{C})$, and so $\Gamma$ is pseudo-finite. In Case 3 , one of $l, m$ is equal to 2 and $\lambda=0$, so that $\Gamma$ only admits an essential representation to a finite dihedral group and hence is pseudo-finite.

(2) In this case there exists an essential representation $\Gamma \rightarrow \mathbb{Z}_{l m n}$ given by $x \mapsto m n$, $y \mapsto \ln$. Thus there exists a homomorphism $\Gamma \rightarrow \operatorname{PSL}(2, \mathbb{C})$ given by $x \mapsto[X]$, $y \mapsto[Y]$ where $X, Y$ are the matrices defined at (4). Since $\operatorname{tr} X Y=2 \cos (\pi / l+\pi / m)$, 
the only essential representations of $\Gamma$ are to elementary subgroups of $\operatorname{PSL}(2, \mathbb{C})$, and so $\Gamma$ is pseudo-elementary. Since $\Gamma$ has an essential representation to a cyclic group, [12, Proposition 1] implies that $\Gamma$ is not pseudo-finite.

(3) Let $\bar{\Gamma}$ be the (ordinary) triangle group $\bar{\Gamma}=\left\langle\bar{x}, \bar{y} \mid \bar{x}^{l / \alpha_{1}}=\bar{y}^{m / \beta_{1}}=(\bar{x} \bar{y})^{n}=1\right\rangle$. Since $\kappa<1$, there exists an essential representation $\bar{\phi}: \bar{\Gamma} \rightarrow \operatorname{PSL}(2, \mathbb{C})$ given by $\bar{x} \mapsto[\bar{X}], \bar{y} \mapsto[\bar{Y}]$ (for some projective matrices $[\bar{X}],[\bar{Y}]$ ) with non-elementary image. Let $[X]$ be an $\left(\alpha_{1}\right)$ th $\operatorname{root}$ of $[\bar{X}]$ and let $[Y]$ be a $\left(\beta_{1}\right)$ th $\operatorname{root}$ of $[\bar{Y}]$ in $\operatorname{PSL}(2, \mathbb{C})$. Then the map $\phi: \Gamma \rightarrow \operatorname{PSL}(2, \mathbb{C})$ given by $x \mapsto[X], y \mapsto[Y]$ defines a faithful (and hence essential) representation with non-elementary image; thus $\Gamma$ is not pseudoelementary.

Theorem 5 completely deals with the group $\Gamma$ when $k=1$, since by passing to another generating pair (if necessary) we may assume that $\alpha_{1}\left|l, \beta_{1}\right| m$. The pseudo-finite groups $\Gamma$ with $k=1$ were classified in [12]. For the following corollary we temporarily ignore the ordering $l \leqslant m$.

Corollary 6. Let $k=1$ and suppose that $\alpha_{1}\left|l, \beta_{1}\right| m$. Then $\Gamma$ is pseudo-elementary but not pseudo-finite if and only if $(l, m, n)$ is equal to $\left(2 \alpha_{1}, 3 \beta_{1}, 6\right),\left(3 \alpha_{1}, 2 \beta_{1}, 6\right),\left(2 \alpha_{1}, 6 \beta_{1}, 3\right)$, $\left(6 \alpha_{1}, 2 \beta_{1}, 3\right),\left(3 \alpha_{1}, 6 \beta_{1}, 2\right),\left(6 \alpha_{1}, 3 \beta_{1}, 2\right),\left(2 \alpha_{1}, 4 \beta_{1}, 4\right),\left(4 \alpha_{1}, 2 \beta_{1}, 4\right),\left(4 \alpha_{1}, 4 \beta_{1}, 2\right)$, or $\left(3 \alpha_{1}, 3 \beta_{1}, 3\right)$.

In the situation of Corollary 6 , if $\alpha_{1}=\beta_{1}=1$ then $\Gamma$ is a Euclidean triangle group, and so is soluble. In all other cases $1 / l+1 / m+1 / n<1$ so that, by [1, Theorem B], $\Gamma$ contains a non-abelian free subgroup. These observations, together with the fact that $\Gamma$ contains a non-abelian free subgroup if it is not pseudoelementary, form a special case of [8, Theorem 6] (or [9, Theorem 2] or [13, Theorem 7.3.2.2]).

From now on we assume that $k \geqslant 2$.

\section{The case $n \geqslant 3$}

The following argument is due to Vinberg and Kaplinsky [12]. The group $\Gamma$ admits an essential representation to $\operatorname{PSL}(2, \mathbb{C})$ if and only if $\lambda$ is a root of $(2)$, where $(r, n)=1$. Varying the value of $r$, we obtain $\phi(n)$ different equations of this form with the fixed polynomial $\tau_{w}(\lambda)$ on the left-hand side. These equations have no common roots. Let $p$ denote the total number of roots of these equations, counting multiplicities, that are repeated; let $q$ denote the total number of roots that are not repeated; and let $N$ denote the total number of different roots, not counting multiplicities. Each of the unrepeated roots is different and so $q \leqslant N$. Each repeated root is a root of the derivative $\tau_{w}^{\prime}(\lambda)$ and this has $k-1$ roots (counting multiplicities), so that $p \leqslant k-1$. The total number of roots (counting multiplicities) of the $\phi(n)$ equations is $k \phi(n)$. Thus $k \phi(n)=p+q \leqslant(k-1)+N$, or equivalently

$$
k(\phi(n)-1) \leqslant N-1 .
$$


If $\Gamma$ is pseudo-elementary then in each case it is possible to calculate the possible values of $\operatorname{tr} X Y$ and thus the value of $N$. If $\phi(n) \geqslant 2$ then inequality (6) gives upper bounds on $k$ and $\phi(n)$.

We first consider the non-exceptional cases.

Theorem 7. Suppose $\Gamma$ is non-exceptional and that one of the following holds:

(i) $\phi(n) \geqslant 4$ and $k \geqslant 1$;

(ii) $\phi(n)=2$ and $k \geqslant 3$;

(iii) $\phi(n)=2, k=2$ and $l \geqslant 3$.

Then $\Gamma$ is not pseudo-elementary.

Proof. Suppose that $\Gamma$ is pseudo-elementary. Since $\Gamma$ is non-exceptional we only need consider Cases 2 and 3 of Theorem 1, so that $N \leqslant 3$ in (i) and (ii), and $N \leqslant 2$ in (iii). We thus obtain a contradiction to (6) and hence $\Gamma$ is not pseudo-elementary.

Theorem 7 is a special case of the proof of [8, Theorem 7], which was obtained by different methods. That theorem is reproduced as [9, Theorem 3] and [13, Theorem 7.3.2.3]; we also refer the reader to the proofs of those theorems in [9], [13] when taken in combination with the remarks of $[6, \mathrm{p}$. 117] concerning the proof of $[8$, Theorem 7].

Consider then the case $k=2$. Let

$$
X=\left(\begin{array}{cc}
e^{\pi i / l} & 1 \\
0 & e^{-\pi i / l}
\end{array}\right), \quad Y=\left(\begin{array}{cc}
e^{\pi i / m} & 0 \\
t & e^{-\pi i / m}
\end{array}\right)
$$

Then

$$
\operatorname{tr} X=2 \cos (\pi / l), \quad \operatorname{tr} Y=2 \cos (\pi / m)
$$

and

$$
\lambda:=\operatorname{tr} X Y=t+2 \cos (\pi / l+\pi / m) .
$$

Noting that

$$
X^{\alpha}=\left(\begin{array}{cc}
e^{\alpha \pi i / l} & \frac{\sin (\alpha \pi / l)}{\sin (\pi / l)} \\
0 & e^{-\alpha \pi i / l}
\end{array}\right), \quad Y^{\beta}=\left(\begin{array}{cc}
e^{\beta \pi i / m} & 0 \\
\frac{\sin (\beta \pi / m)}{\sin (\pi / m)} t & e^{-\beta \pi i / m}
\end{array}\right)
$$

we may calculate that

$$
\operatorname{tr} X^{\alpha_{1}} Y^{\beta_{1}} X^{\alpha_{2}} Y^{\beta_{2}}=A t^{2}+B t+C,
$$

where 


$$
\begin{aligned}
A= & \frac{\sin \left(\alpha_{1} \pi / l\right) \sin \left(\beta_{1} \pi / m\right) \sin \left(\alpha_{2} \pi / l\right) \sin \left(\beta_{2} \pi / m\right)}{\sin ^{2}(\pi / l) \sin ^{2}(\pi / m)}, \\
B= & \frac{1}{\sin (\pi / l) \sin (\pi / m)}\left(\sin \left(\frac{\left(\alpha_{1}+\alpha_{2}\right) \pi}{l}\right) \sin \left(\frac{\left(\beta_{1}+\beta_{2}\right) \pi}{m}\right)\right. \\
& \left.-4 \sin \left(\alpha_{1} \pi / l\right) \sin \left(\beta_{1} \pi / m\right) \sin \left(\alpha_{2} \pi / l\right) \sin \left(\beta_{2} \pi / m\right)\right), \\
C= & 2 \cos \left(\frac{\left(\alpha_{1}+\alpha_{2}\right) \pi}{l}+\frac{\left(\beta_{1}+\beta_{2}\right) \pi}{m}\right) .
\end{aligned}
$$

Theorem 8. Suppose that $l=2, m \geqslant 6$ and that $\phi(n)=2, k=2$. Then $\Gamma$ is pseudoelementary if and only if one of the following holds:

(1) $n=3$ and $\left\{\beta_{1}, \beta_{2}\right\}=\{m / 2, m / 6\},\{m / 2,5 m / 6\}$, where $m$ is divisible by 6 ;

(2) $n=4$ and $\left\{\beta_{1}, \beta_{2}\right\}=\{m / 2, m / 4\},\{m / 2,3 m / 4\}$, where $m$ is divisible by 4 ;

(3) $n=6$ and $\left\{\beta_{1}, \beta_{2}\right\}=\{m / 2, m / 3\},\{m / 2,2 m / 3\}$, where $m$ is divisible by 6 .

Proof. Suppose that $\Gamma$ is pseudo-elementary. Setting $t=\lambda-2 \cos (\pi / l+\pi / m)$, we find that the $\phi(n)$ equations of the form (2) are

$$
\begin{aligned}
& A t^{2}+B t+C=D, \\
& A t^{2}+B t+C=-D
\end{aligned}
$$

where $D=2 \cos (r \pi / n)$ with $(r, n)=1$ and (using $(7))$

$$
\begin{aligned}
& A=\frac{\sin \left(\beta_{1} \pi / m\right) \sin \left(\beta_{2} \pi / m\right)}{\sin ^{2}(\pi / m)}, \quad B=\frac{-4 \sin \left(\beta_{1} \pi / m\right) \sin \left(\beta_{2} \pi / m\right)}{\sin (\pi / m)}, \\
& C=-2 \cos \left(\left(\beta_{1}+\beta_{2}\right) \pi / m\right) .
\end{aligned}
$$

The possible roots of (8), (9) are $t_{i}=\lambda_{i}+2 \sin (\pi / m)$ for $i=0,1,2$, where $\lambda_{0}=0$, $\lambda_{1}=2 \sin (\pi / m), \lambda_{2}=-2 \sin (\pi / m)$; that is, $t_{0}=2 \sin (\pi / m), t_{1}=0, t_{2}=4 \sin (\pi / m)$. Since $D \neq 0$ the equations (8), (9) have no common roots, and so one of them must have a repeated root. The repeated root is $t=-B / 2 A=t_{0}$. By replacing $r$ by $(n-r)$ (if necessary) we may assume that (8) has the repeated root $t_{0}$. This occurs if and only if $B^{2}=4 A(C-D)$, or equivalently

$$
\cos \left(\left(\beta_{1}-\beta_{2}\right) \pi / m\right)=-\cos (r \pi / n) .
$$

Since $t_{1}, t_{2}$ cannot be repeated roots, one of the roots of (9) must be $t_{1}$, so that we have $C=-D$, or equivalently

$$
\cos \left(\left(\beta_{1}+\beta_{2}\right) \pi / n\right)=\cos (r \pi / n) .
$$


The simultaneous equations (11), (12) hold if and only if $\cos \left(\beta_{1} \pi / m\right) \cos \left(\beta_{2} \pi / m\right)=0$, i.e. if and only if at least one of $\beta_{1} / m, \beta_{2} / m$ is equal to $1 / 2$.

Let $\left\{\beta_{1} / m, \beta_{2} / m\right\}=\{1 / 2, \theta / m\}$, where $1 \leqslant \theta \leqslant m-1$. Then (12) is equivalent to $\cos (r \pi / n)=-\sin (\theta \pi / m)$. When $n=3$ we have $\theta / m=1 / 6$ or $5 / 6$, if $n=4$ we have $\theta / m=1 / 4$ or $3 / 4$, if $n=6$ we have $\theta / m=1 / 3$ or $2 / 3$, and the proof is complete.

Theorem 8 is a special case of the proof of [8, Theorem 7] taken together with an observation of Hennig [14]: see [6, p. 117]. Note that in each case $\Gamma$ maps onto $\left\langle x, y \mid x^{2}=y^{m / 2}=1\right\rangle$ and so contains a non-abelian free subgroup (since $m \geqslant 6$ ).

We now consider the exceptional cases.

Theorem 9. Suppose $\Gamma$ is exceptional and that $n \geqslant 3, k \geqslant 2$. Then $\Gamma$ is pseudoelementary if and only if $\Gamma$ is one of the following groups:

- $\Gamma_{1}=\left\langle x, y \mid x^{2}=y^{3}=\left(x y x y^{2}\right)^{3}=1\right\rangle$;

$\cdot \Gamma_{2}=\left\langle x, y \mid x^{2}=y^{3}=\left(x y x y x y^{2}\right)^{3}=1\right\rangle$;

- $\Gamma_{3}=\left\langle x, y \mid x^{2}=y^{3}=\left(x y x y x y x y^{2}\right)^{3}=1\right\rangle$;

- $\Gamma_{4}=\left\langle x, y \mid x^{2}=y^{4}=\left(x y x y^{2}\right)^{4}=1\right\rangle$;

- $\Gamma_{5}=\left\langle x, y \mid x^{2}=y^{4}=\left(x y x y^{2} x y^{2} x y^{2}\right)^{4}=1\right\rangle$;

- $\Gamma_{6}=\left\langle x, y \mid x^{3}=y^{3}=\left(x y x y^{2}\right)^{3}=1\right\rangle$;

$\cdot \Gamma_{7}=\left\langle x, y \mid x^{4}=y^{4}=\left(x y^{2} x^{2} y^{2}\right)^{4}=1\right\rangle$.

Proof. If $\Gamma$ is pseudo-finite then by [12, Proposition 5], $\Gamma$ is one of the groups $\Gamma_{1}, \Gamma_{2}$. Assume then that $\Gamma$ is not pseudo-finite.

For each pair $(l, m),[12$, Table 3$]$ lists the possible values of $\lambda$ that correspond to essential representations to $A_{4}, S_{4}$, or $A_{5}$. In addition to these, we must consider the values of $\lambda$ in Cases 2 and 3 of Theorem 1. (Note that $\lambda=0$ may fall both into Case 1 and Case 2.) For each pair $(l, m)$ we therefore know the total number of roots $N$ of (2) that correspond to essential representations to elementary subgroups of $\operatorname{PSL}(2, \mathbb{C})$. (Namely, $N=11$ if $(l, m)=(2,3), N=7$ if $(l, m)=(2,5), N=6$ if $(l, m)=(3,3)$ or $(3,5), N=5$ if $(l, m)=(2,4), N=4$ if $(l, m)=(3,4)$ or $(5,5)$, and $N=3$ if $(l, m)=(4,4)$.) Inequality $(6)$ then provides an upper bound $K$ on $k$ and an upper bound on $\phi(n)$. We obtain that $(l, m: \phi(n), K)$ is equal to $(2,3: 2,10)$, $(2,3: 4,3),(2,3: 6,2),(2,4: 2,4),(2,5: 4,2),(2,5: 2,6),(3,3: 2,5),(3,4: 2,3)$, $(3,5: 2,5),(4,4: 2,2)$, or $(5,5: 2,3)$.

Since $\Gamma$ is not pseudo-finite the roots corresponding to Case 2 of Theorem 1 must occur, and thus $\Gamma$ admits an essential representation to a cyclic group. We can therefore eliminate cases where no such representation is possible. By [12, Proposition 2] if any of the following hold then $\Gamma$ does not admit an essential cyclic representation:

$\cdot l, m$ are odd and $n$ is even;

- $p \nmid l, p \nmid m, p \mid n$, where $p \geqslant 3$ is prime; 
- $p^{2} \nmid l, p^{2} \nmid m, p^{2} \mid n$, where $p \geqslant 3$ is prime;

$\cdot l=2, m$ is odd, and $n, k$ are of the same parity.

These eliminations reduce us to the cases when $(l, m, n ; K)$ is equal to $(2,4,4 ; 4)$, $(2,5,5 ; 2), \quad(2,5,10 ; 2), \quad(3,3,3 ; 5), \quad(3,4,3 ; 3), \quad(3,4,4 ; 3), \quad(3,4,6 ; 3), \quad(3,5,3 ; 5)$, $(4,4,4 ; 2)$, or $(2,3,3 ; 10)$ where $k$ is even, or $(2,3,6 ; 10)$ where $k$ is odd. In these cases an exhaustive computer search (using Maple) for words $w(x, y)$ such that the roots of (2) all correspond to essential representations to elementary subgroups of $\operatorname{PSL}(2, \mathbb{C})$ was run. This revealed that $\Gamma$ is pseudo-elementary (but not pseudo-finite) precisely in the cases $\Gamma_{3}, \ldots, \Gamma_{7}$.

In the case $(l, m, n) \neq(2,3,3)$, Theorem 9 is a special case of the proof of [8, Theorem 7]. (See also the proofs of [9, Theorem 3] or [13, Theorem 7.3.2.3] together with the remarks of [6, p. 117].) In [8, Theorem 7] it is further shown that $\Gamma_{4}, \Gamma_{5}, \Gamma_{6}, \Gamma_{7}$ all contain non-abelian free subgroups. In the case $(l, m, n)=(2,3,3)$, Theorem 9 was given in [8, p. 107] (see also [9, Theorem 4]) or [13, Theorem 7.3.2.4], where it was further shown that $\Gamma_{1}$ is infinite and virtually abelian, $\Gamma_{2}$ is finite of order 1440 , and $\Gamma_{3}$ is infinite and soluble.

From now on we assume that $n=2$.

\section{The non-exceptional cases with $n=2, k=2$}

Consider first the case $l=2$.

Theorem 10. Suppose that $l=n=2, m \geqslant 6$ and that $k=2$. Then $\Gamma$ is pseudoelementary if and only if $m$ is even and $\beta_{2}=m / 2-\beta_{1}, 3 m / 2-\beta_{1}$, or $\beta_{1} \pm m / 2$.

Proof. Suppose that $\Gamma$ is pseudo-elementary. Setting $t=\lambda-2 \cos (\pi / l+\pi / m)$, equation (2) becomes

$$
A t^{2}+B t+C=0
$$

where $A, B, C$ are given by (10). The possible roots of (13) are $t_{0}=2 \sin (\pi / m)$, $t_{1}=0, t_{2}=4 \sin (\pi / m)$.

Now $C=0$ if and only if $\beta_{2}=m / 2-\beta_{1}$ or $\beta_{2}=3 m / 2-\beta_{1}$ (where $m$ is even). In this case (13) has roots $t_{1}, t_{2}$, and so $\Gamma$ is pseudo-elementary. Suppose then that $C \neq 0$. The sum of the roots of $(13)$ is $-B / A=4 \sin (\pi / m)$, and since $t_{1}$ is not a root the only root is $t_{0}$. The product of the roots is $C / A$ so that

$$
4 \sin ^{2}(\pi / m)=\frac{-2 \cos \left(\left(\beta_{1}+\beta_{2}\right) \pi / m\right) \sin ^{2}(\pi / m)}{\sin \left(\beta_{1} \pi / m\right) \sin \left(\beta_{2} \pi / m\right)},
$$

which holds if and only if $\beta_{2}=\beta_{1} \pm m / 2$ (where $m$ is even). 
By [16, Propositions 8, 9], the group $\Gamma$ contains a non-abelian free subgroup unless $\Gamma=\left\langle x, y \mid x^{2}=y^{6}=\left(x y x y^{2}\right)^{2}=1\right\rangle$ or $\Gamma=\left\langle x, y \mid x^{2}=y^{6}=\left(x y x y^{4}\right)^{2}=1\right\rangle$, in which case $\Gamma$ is infinite and soluble.

Consider now the cases where $l \geqslant 3$. Suppose that $\Gamma$ is pseudo-elementary. Setting $t=\lambda-2 \cos (\pi / l+\pi / m)$, equation (2) becomes (13) where $A, B, C$ are given by (10). Since $l, m \geqslant 3$, Case 3 of Theorem 1 cannot occur, and so the possible roots of (13) are $t_{1}=0, t_{2}=4 \sin (\pi / l) \sin (\pi / m)$. By replacing $w(x, y)$ with the equivalent word $w\left(x, y^{-1}\right)$ (if necessary) we may assume that $t_{1}$ occurs as a root. This occurs if and only if $C=0$, i.e. if and only if

$$
\cos \left(\frac{\left(\alpha_{1}+\alpha_{2}\right) \pi}{l}+\frac{\left(\beta_{1}+\beta_{2}\right) \pi}{m}\right)=0 .
$$

The second root of (13) is therefore $t=-B / A$, i.e.

$$
t=4 \sin (\pi / l) \sin (\pi / m)-\frac{\sin (\pi / l) \sin (\pi / m) \sin \left(\left(\alpha_{1}+\alpha_{2}\right) \pi / l\right) \sin \left(\left(\beta_{1}+\beta_{2}\right) \pi / m\right)}{\sin \left(\alpha_{1} \pi / l\right) \sin \left(\beta_{1} \pi / m\right) \sin \left(\alpha_{2} \pi / l\right) \sin \left(\beta_{2} \pi / m\right)} .
$$

Proposition 11. The roots of (13) are $t_{1}, t_{2}$ if and only if $\left(\alpha_{2}, \beta_{2}\right)$ is equal to $\left(l-\alpha_{1}, m / 2-\beta_{1}\right),\left(l-\alpha_{1}, 3 m / 2-\beta_{1}\right)$ (where $m$ is even) or $\left(l / 2-\alpha_{1}, m-\beta_{1}\right)$, $\left(3 l / 2-\alpha_{1}, m-\beta_{1}\right)$ (where l is even).

Proof. By the above, $t_{1}$ is a root if and only if (14) holds, and (because of (15)) $t_{2}$ is the other root if and only if $\sin \left(\left(\alpha_{1}+\alpha_{2}\right) \pi / l\right) \sin \left(\left(\beta_{1}+\beta_{2}\right) \pi / m\right)=0$; that is, if and only if $\alpha_{1}+\alpha_{2}=l$ or $\beta_{1}+\beta_{2}=m$. If $\alpha_{1}+\alpha_{2}=l$ then (14) is satisfied if and only if $m$ is even and $\beta_{1}+\beta_{2}$ equals $m / 2$ or $3 m / 2$. If $\beta_{1}+\beta_{2}=m$ then (14) is satisfied if and only if $l$ is even and $\alpha_{1}+\alpha_{2}=l / 2$ or $3 l / 2$.

Hence $\Gamma$ is pseudo-elementary in these cases.

Note that in each case $\Gamma$ maps homomorphically onto $\left\langle x, y \mid x^{\alpha_{1}}=y^{m}=1\right\rangle$ and onto $\left\langle x, y \mid x^{l}=y^{\beta_{1}}=1\right\rangle$, and so contains a non-abelian free subgroup unless $\alpha_{1}=\beta_{1}=1$. In this case, by [1, Theorem B], $\Gamma$ contains a non-abelian free subgroup unless $l=3, m=6$; but then $\Gamma$ maps onto $\left\langle x, y \mid x^{3}=y^{2}=1\right\rangle$, and so contains a non-abelian free subgroup.

Suppose then the roots of (13) are $t_{1}, t_{1}$. This occurs if and only if (14) and (16) (below) both hold:

$$
\sin \left(\frac{\left(\alpha_{1}+\alpha_{2}\right) \pi}{l}\right) \sin \left(\frac{\left(\beta_{1}+\beta_{2}\right) \pi}{m}\right)=4 \sin \left(\frac{\alpha_{1} \pi}{l}\right) \sin \left(\frac{\beta_{1} \pi}{m}\right) \sin \left(\frac{\alpha_{2} \pi}{l}\right) \sin \left(\frac{\beta_{2} \pi}{m}\right) .
$$

There appear to be very many solutions to these equations. Let us assume that the denominators $l, m$ are in their lowest form; that is, $\left(\alpha_{1}, \alpha_{2}, l\right)=\left(\beta_{1}, \beta_{2}, m\right)=1$. It is easy to verify that 
$\mathrm{A}(s): l=m=8 s$,

$$
\left\{\left\{\alpha_{1}, \alpha_{2}\right\},\left\{\beta_{1}, \beta_{2}\right\}\right\}=\{\{p, p+l / 2\},\{l / 4-p, 3 l / 4-p\}\}, \quad(p, l)=1 ;
$$

$\mathrm{B}(s): l=4 s+2, m=2 l$,

$$
\left(\left\{\alpha_{1}, \alpha_{2}\right\},\left\{\beta_{1}, \beta_{2}\right\}\right)=(\{p, l / 2+p\},\{l / 2-2 p, 3 l / 2-2 p\}), \quad(p, l / 2)=1 ;
$$

define solutions for all $s \geqslant 1$. A computer search (using Maple) shows that each solution with $2 \leqslant l \leqslant m \leqslant 80$ either takes the form $\mathrm{A}(s)$ or $\mathrm{B}(s)$, or takes one of the following forms:

$\mathrm{E} 1: l=10, m=15$,

$\left(\left\{\alpha_{1}, \alpha_{2}\right\},\left\{\beta_{1}, \beta_{2}\right\}\right),\left(\left\{l-\alpha_{1}, l-\alpha_{2}\right\},\left\{m-\beta_{1}, m-\beta_{2}\right\}\right)=(\{1,8\},\{2,7\})$

or $(\{3,4\},\{1,11\})$;

$\mathrm{E} 2: l=12, m=12$,

$\left\{\left\{\alpha_{1}, \alpha_{2}\right\},\left\{\beta_{1}, \beta_{2}\right\}\right\},\left\{\left\{l-\alpha_{1}, l-\alpha_{2}\right\},\left\{m-\beta_{1}, m-\beta_{2}\right\}\right\}=\{\{1,10\},\{2,5\}\} ;$

E3: $l=14, m=14$,

$\left\{\left\{\alpha_{1}, \alpha_{2}\right\},\left\{\beta_{1}, \beta_{2}\right\}\right\},\left\{\left\{l-\alpha_{1}, l-\alpha_{2}\right\},\left\{m-\beta_{1}, m-\beta_{2}\right\}\right\}=\{\{1,9\},\{3,8\}\}$,

$\{\{1,12\},\{3,5\}\}$, or $\{\{1,11\},\{4,5\}\}$;

$\mathrm{E} 4: l=15, m=30$,

$\left(\left\{\alpha_{1}, \alpha_{2}\right\},\left\{\beta_{1}, \beta_{2}\right\}\right),\left(\left\{l-\alpha_{1}, l-\alpha_{2}\right\},\left\{m-\beta_{1}, m-\beta_{2}\right\}\right)=(\{1,7\},\{3,26\})$,

$(\{1,13\},\{8,9\}),(\{2,11\},\{3,16\})$, or $(\{4,7\},\{2,21\})$;

E5: $l=20, m=80$,

$\left(\left\{\alpha_{1}, \alpha_{2}\right\},\left\{\beta_{1}, \beta_{2}\right\}\right),\left(\left\{l-\alpha_{1}, l-\alpha_{2}\right\},\left\{m-\beta_{1}, m-\beta_{2}\right\}\right)=(\{5,6\},\{5,71\}) ;$

E6: $l=39, m=78$,

$\left(\left\{\alpha_{1}, \alpha_{2}\right\},\left\{\beta_{1}, \beta_{2}\right\}\right),\left(\left\{l-\alpha_{1}, l-\alpha_{2}\right\},\left\{m-\beta_{1}, m-\beta_{2}\right\}\right)=(\{10,10\},\{3,74\}) ;$

E7: $l=40, m=75$,

$\left(\left\{\alpha_{1}, \alpha_{2}\right\},\left\{\beta_{1}, \beta_{2}\right\}\right),\left(\left\{l-\alpha_{1}, l-\alpha_{2}\right\},\left\{m-\beta_{1}, m-\beta_{2}\right\}\right)=(\{3,33\},\{17,28\}) ;$

$\mathrm{E} 8: l=55, m=66$,

$\left(\left\{\alpha_{1}, \alpha_{2}\right\},\left\{\beta_{1}, \beta_{2}\right\}\right),\left(\left\{l-\alpha_{1}, l-\alpha_{2}\right\},\left\{m-\beta_{1}, m-\beta_{2}\right\}\right)=(\{2,28\},\{11,52\}) ;$

E9: $l=75, m=80$,

$\left(\left\{\alpha_{1}, \alpha_{2}\right\},\left\{\beta_{1}, \beta_{2}\right\}\right),\left(\left\{l-\alpha_{1}, l-\alpha_{2}\right\},\left\{m-\beta_{1}, m-\beta_{2}\right\}\right)=(\{2,43\},\{29,43\})$.

We have not been able to classify all solutions. 
Acknowledgement. The author would like to thank Jim Howie for helpful discussions relating to this work, and the referee, whose comments highlighted a mistake in the original version of this paper.

\section{References}

[1] G. Baumslag, J. W. Morgan and P. B. Shalen. Generalized triangle groups. Math. Proc. Cambridge Philos. Soc. 102 (1987), 25-31.

[2] V. V. Benyash-Krivets. On free subgroups of certain generalized triangle groups. Dokl. Nat. Akad. Nauk. Belarusi 47 (2003), 14-17.

[3] O. A. Barkovich and V. V. Benyash-Krivets. On the Tits alternative for generalized triangular groups of $(2,6,2)$ type. Dokl. Nat. Akad. Nauk. Belarusi 48 (2003), 28-33.

[4] S. Boyer. On proper powers in free products and Dehn surgery. J. Pure Appl. Algebra 51 (1988), 217-229.

[5] H. S. M. Coxeter and W. O. J. Moser. Generators and relations for discrete groups (Springer-Verlag, 1972).

[6] M. Edjvet, J. Howie, G. Rosenberger and R. M. Thomas. Finite generalized tetrahedron groups with a high-power relator. Geom. Dedicata 94 (2002), 111-139.

[7] B. Fine, J. Howie and G. Rosenberger. One-relator quotients and free products of cyclics. Proc. Amer. Math. Soc. 102 (1988), 249-254.

[8] B. Fine, F. Levin and G. Rosenberger. Free subgroups and decompositions of one-relator products of cyclics. I. The Tits alternative. Arch. Math. (Basel) 50 (1988), 97-109.

[9] B. Fine, F. Roehl and G. Rosenberger. The Tits alternative for generalized triangle groups. In Groups-Korea '98 (Pusan), (de Gruyter, 2000), pp. 95-131.

[10] B. Fine and G. Rosenberger. A note on generalized triangle groups. Abh. Math. Sem. Univ. Hamburg 56 (1986), 233-244.

[11] R. Ree and N. S. Mendelsohn. Free subgroups of groups with a single defining relation. Arch. Math. (Basel) 19 (1968), 577-580.

[12] E. B. Vinberg and K. V. Kaplinsky. Pseudo-finite generalized triangle groups. In Groups: topological, combinatorial and arithmetic aspects, London Math. Soc. Lecture Note Ser. 311 (Cambridge University Press, 2004), pp. 564-587.

[13] B. Fine and G. Rosenberger. Algebraic generalizations of discrete groups: a path to combinatorial group theory through one-relator products (Marcel Dekker, 1999).

[14] D. Hennig. Titsalternative für verallgemeinerte Dreiecksgruppen. Diplomarbeit. Universität Dortmund (1999).

[15] J. Howie and G. Williams. The Tits alternative for certain generalized triangle groups of type $(2, m, 2)$. Geom. Dedicata, to appear.

[16] G. Rosenberger. On free subgroups of generalized triangle groups. Algebra i Logika 28 (1989), 227-240.

Received 30 August, 2005; revised 11 January, 2006

Gerald Williams, Institute of Mathematics, Statistics, and Actuarial Science, University of Kent, Canterbury, Kent CT2 7NF, U.K.

E-mail: g.williams@kent.ac.uk 